\title{
Detection of T Wave Alternans in Non-stationary Noise: a GLRT Approach
}

\author{
JP Martínez, S Olmos \\ GTC, Aragon Institute of Engineering Research (I3A), University of Zaragoza, Aragon, Spain
}

\begin{abstract}
$T$ wave alternans (TWA) has been proposed as a marker for cardiac instability and high risk of malignant ventricular arrhythmias. In this work, we analyze a Generalized Likelihood Ratio Test (GLRT) approach to TWA detection. We used several noise models considering Gaussian and Laplacian distributions as well as three stationarity degrees. Another novelty of the proposed detectors is the use of the data from the P wave. The GLRT detectors for all the models were derived and implemented. Their detection performance was evaluated using real ECG signals with simulated TWA, showing that models accounting for the non-stationarity of the noise obtained best results. We also found that Laplacian-based detectors outperformed those based on Gaussian noise assumption.
\end{abstract}

\section{Introduction}

Electrical T-wave alternans (TWA) is defined as a consistent fluctuation in the repolarization morphology which repeats on an every-other-beat basis. It has been documented in a wide range of experimental and clinical situations, such as long QT syndrome, myocardial ischemia and infarction, coronary artery occlusion, Printzmetal angina and several other pathological conditions.

Although visible TWA is an infrequent phenomenon, computerized analysis of digital ECG recordings allowed in recent years the identification of subtle and non-visible (microvolt) TWA, much more common than visible TWA. Recently, several studies showed that TWA is related to cardiac instability and high risk for malignant ventricular arrhythmias and sudden cardiac death [1]. Thus the importance of deriving robust and sensitive methods for detecting TWA in ECG signals.

Several methods for TWA detection have been proposed. Most of them are based on the well-known problem of spectral estimation. The most widely used methods are the spectral method (SM) and the complex demodulation (CD). The SM [1] used the FFT to analyze the frequency component 0.5 cycles/beat over the aligned ST$\mathrm{T}$ complexes. In the $\mathrm{CD}$ approach [2], the alternant component is demodulated and low-pass filtered to obtain a continuous beat-to-beat alternans measure.
In a previous work [3], we showed that both SM and CD can be seen as GLRT TWA detectors "matched" to a given episode shape and duration immersed in Gaussian noise. We also developed a similar GLRT detector for Laplacian noise, which is a more realistic assumption for the noise present in TWA. In both cases, the noise variance was assumed constant and known. However, in real ECG conditions, noise variance is unknown and usually nonstationary, with the consequent degradation in detection performance.

In this paper, we derive detectors for non-stationary noise models with Gaussian as well as Laplacian statistics. We also consider auxiliary data from the $\mathrm{P}$ wave, which participates in the estimation of noise parameters. The models and the derived GLRT detectors are presented in Section 2, where we also describe the simulation study used to assess the detection performance. The results are given and discussed in Sections 3 and 4, while in Section 5 we expose the conclusions of the work.

\section{Methods}

\subsection{Signal and noise models}

In most of the published methods for TWA analysis, consecutive ST-T complexes are extracted, aligned and put together into an ST-T matrix $\mathbf{X}$, whose $i$ :th column contains the $i$ :th beat's ST-T complex ( $N_{T}$ samples). Equivalently, the $j$ :th row is formed by a beat-to-beat amplitude series containing the samples with the same phase within the repolarization interval. If there is no available prior knowledge about the episode shape, a reasonable assumption is to consider the alternans episode to be constant within an analysis window of $L$ beats. Then, the $N_{T} \times L$ ST-T matrix $\mathbf{X}$ can be modeled as

$$
\mathbf{X}=\mathbf{t} \mathbf{1}^{T}+\mathbf{a} \mathbf{e}_{a}^{T}+\mathbf{W}
$$

where $\mathbf{t}=\left[t[0], \ldots, t\left[N_{T}-1\right]\right]^{T}$ is the periodic T-wave, $\mathbf{a}=\left[a[0], \ldots, a\left[N_{T}-1\right]\right]^{T}$ is the alternans waveform, $\mathbf{1}$ is an all-ones $L \times 1$ vector, and $\mathbf{e}_{a}$ is the $L \times 1$ alternant vector $\left[(-1)^{0}, \ldots,(-1)^{L-1}\right]^{T}$. All the other non-desired components can be grouped into the noise matrix $\mathbf{W}$.

However, in ECG analysis, we have access to additional available data, such as $\mathrm{P}$ wave, where TWA does not affect the signal. We propose in this work to include these data in 
the signal model. That information can be used to improve the noise power estimation, and consequently, the TWA detection performance. The signal model is then

$$
\mathbf{X}=\left[\begin{array}{c}
\mathbf{p} \\
\mathbf{t}
\end{array}\right] \mathbf{1}^{T}+\left[\begin{array}{c}
\mathbf{0} \\
\mathbf{a}
\end{array}\right] \mathbf{e}_{a}^{T}+\mathbf{W}
$$

with $\mathbf{p}=\left[p[0], \ldots, p\left[N_{P}-1\right]\right]^{T}$ the periodic P-wave. The data matrix $\mathbf{X}$ is now a $N \times L$ matrix (where $N=N_{P}+N_{T}$ ) whose columns are the concatenation of $\mathrm{P}$ wave and ST-T complex samples in $L$ consecutive beats. The $n$ :th row, i.e. the beat-to-beat series composed of the $n$ :th sample of each complex, is then

$$
x_{l}[n]= \begin{cases}p[n]+w_{l}[n], & 0 \leq n<N_{P} \\ t\left[n-N_{p}\right]+a\left[n-N_{p}\right](-1)^{l}+w_{l}[n], & N_{p} \leq n<N\end{cases}
$$

for $l=0, \ldots, L-1$. Subtracting an estimation of the periodic component (the mean of each row) or using a detrending filter, the constant terms corresponding to the repetitive $\mathrm{P}$ / ST-T can be canceled. Thus, the model for the observed detrended series reduces to

$$
x_{l}^{\prime}[n]= \begin{cases}w_{l}[n], & 0 \leq n<N_{P} \\ a\left[n-N_{p}\right](-1)^{l}+w_{l}[n], & N_{p} \leq n<N\end{cases}
$$

To simplify the notation we will use $y_{l}[n]$ for the demodulated detrended series $x_{l}^{\prime}[n](-1)^{n}$, and the alternans signal will be denoted as

$$
s[n]=\left\{\begin{array}{cc}
0 & 0 \leq n \leq N_{p}-1 \text { (P wave) } \\
a\left[n-N_{p}\right] & N_{p} \leq n \leq N-1 \text { (ST-T complex) }
\end{array}\right.
$$

The hypothesis testing problem in TWA detection is

$$
\begin{aligned}
& \mathcal{H}_{0}: \mathbf{a}=\mathbf{0} \\
& \mathcal{H}_{1}: \mathbf{a} \neq \mathbf{0} .
\end{aligned}
$$

The noise is generally assumed to be uncorrelated and Gaussian with zero mean and variance $\sigma^{2}$.

In this work, we consider two noise distributions: Gaussian and Laplacian. For each of them, we study three different noise models with different assumptions about the degree of knowledge and stationarity. Model A: the noise is uncorrelated (Gaussian or Laplacian) with zero mean and known variance $\sigma^{2}$. Model $\mathrm{B}$ : the noise is uncorrelated (Gaussian or Laplacian) with zero mean and unknown variance $\sigma^{2}$ and Model $\mathrm{C}$ : the noise is uncorrelated (Gaussian or Laplacian) with zero mean and beat-to-beat changing unknown variance $\sigma_{l}^{2}, l=0, \ldots, L-1$. Model $\mathrm{A}$ is essentially the same approach as in [3] and assumes a constant noise variance for any signal block. Model B assumes a constant noise level within the analysis window, but accounts for slow changes in noise power. The assumption of Model $\mathrm{C}$ is much weaker, and allows beatto-beat changes in the noise variance.

\subsection{GLRT detectors}

The GLRT decides $\mathcal{H}_{1}$ if the likelihood ratio

$$
L_{G}(\mathbf{X})=\frac{p\left(\mathbf{X}^{\prime} ; \hat{\theta}_{\mathbf{1}}, \mathcal{H}_{\mathbf{1}}\right)}{p\left(\mathbf{X}^{\prime} ; \hat{\theta}_{\mathbf{0}}, \mathcal{H}_{\mathbf{0}}\right)}>\gamma,
$$

where $\hat{\theta}_{1}$ and $\hat{\theta}_{0}$ stand for the maximum likelihood estimators (MLE) of the unknown parameters under $\mathcal{H}_{1}$ and $\mathcal{H}_{0}$ respectively.

The number of unknown parameters depends on the assumed model. In model $\mathrm{A}, \hat{\theta}_{1}=\{\hat{\mathbf{a}}\}$ and $\hat{\theta}_{0}=\{\}$. In model $\mathrm{B}, \hat{\theta}_{1}=\left\{\hat{\mathbf{a}}, \hat{\sigma}_{1}^{2}\right\}$ and $\hat{\theta}_{0}=\left\{\hat{\sigma}_{0}^{2}\right\}$. Finally, in model $\mathrm{C}$, we have $\hat{\theta}_{1}=\left\{\hat{\mathbf{a}}, \hat{\sigma}_{i, 1}^{2}\right\}$ and $\hat{\theta}_{0}=\left\{\hat{\sigma}_{i, 0}^{2}\right\}$ for $i=$ $0 \ldots L-1$. The MLE of each unknown parameter depends on the assumed statistical model. In Table 1, the MLE estimators and the GLRT detectors are given for Gaussian and Laplacian pdf. There, med(.) stands for the median operator, $\operatorname{med}\left(\cdot \diamond b_{l}\right)$ for the weighted median with weights $b_{l}$, and

$$
T_{n}(\mathbf{X})= \begin{cases}2 \sum_{0<y_{l}[n] \leq \hat{s}[n]} y_{l}[n] & \text { if } \hat{\mathrm{s}}[\mathrm{n}] \geq 0 \\ -2 \sum_{\hat{s}[n]<y_{l}[n] \leq 0} y_{l}[n] & \text { if } \hat{\mathrm{s}}[\mathrm{n}]<0\end{cases}
$$

i.e., the GLRT for a single alternans series in Laplacian noise, as shown in [3].

Any monotonic function of the GLR is also a GLR Test [4]. Using this property, the detectors in Table 1 can be simplified. The known $\sigma^{2}$ in $T_{G A}(\mathbf{X}$ ) (and $\sigma$ in $T_{L A}(\mathbf{X})$ ) can be dropped out from the test and absorbed by the threshold. In this model, variations of $\sigma$ are not taken into account. From $T_{G B}(\mathbf{X})$ and $T_{L B}(\mathbf{X})$ we can derive the equivalent detectors

$$
\begin{aligned}
T_{G B}^{\prime}(\mathbf{X})=\frac{\frac{1}{N} \sum_{n=0}^{N_{T}-1} \hat{a}^{2}[n]}{\hat{\sigma}_{1}^{2}}=\frac{\frac{1}{N} \sum_{n=0}^{N_{T}-1} \hat{a}^{2}[n]}{\hat{\sigma}_{0}^{2}+\frac{1}{N} \sum_{n=0}^{N_{T}-1} \hat{a}^{2}[n]} \\
T_{L B}^{\prime}(\mathbf{X})=\frac{\frac{\sqrt{2}}{N L} \sum_{n=N_{P}}^{N} T_{n}[n]}{\hat{\sigma}_{1}}=\frac{\frac{\sqrt{2}}{N L} \sum_{n=N_{P}}^{N-1} T_{n}[n]}{\hat{\sigma}_{0}+\frac{\sqrt{2}}{N L} \sum_{n=N_{P}}^{N-1} T_{n}[n]}
\end{aligned}
$$

which are similar to $T_{G A}(\mathbf{X}), T_{L A}(\mathbf{X})$, but estimating $\sigma$ in each data block. As for $T_{G C}$ and $T_{L C}$, they are not easy to simplify. To calculate the MLE under $\mathcal{H}_{1}$, we found a joint maximization of $\hat{a}[n]$ and $\hat{\sigma}_{i, 1}$ (which are coupled). That was solved by following an iterative procedure, departing from an initial estimation of the noise parameters in the $\mathrm{P}$ wave series.

The six TWA detectors corresponding to the six models were implemented and evaluated. All of them share the same preprocessing stage, including QRS detection, baseline wandering, linear filtering (20th-order equiripple 
Table 1. ML estimators and GLRT statistics for the studied noise models.

\begin{tabular}{|c|c|c|c|c|c|c|}
\hline Model & & Gaussian MLE & Gaussian GLRT & & Laplacian MLE & Laplacian GLRT \\
\hline A & $\hat{a}[n]$ & $\frac{1}{L} \sum_{l=0}^{L-1} y_{l}\left[N_{p}+n\right]$ & $T_{G A}(\mathbf{X})=\sum_{n=0}^{N_{T}-1} \frac{\hat{a}^{2}[n]}{\sigma^{2}}$ & $\hat{a}[n]$ & $\operatorname{med}_{1=0}^{\mathrm{L}-1}\left(y_{l}\left[N_{p}+n\right]\right)$ & $T_{L A}(\mathbf{X})=\sum_{n=N_{p}}^{N-1} \frac{T_{n}(\mathbf{X})}{\sigma}$ \\
\hline \multirow{3}{*}{ B } & $\hat{a}[n]$ & $\frac{1}{L} \sum_{l=0}^{L-1} y_{l}\left[N_{p}+n\right]$ & \multirow{3}{*}{$T_{G B}(\mathbf{X})=\frac{\hat{\sigma}_{0}^{2}}{\hat{\sigma}_{1}^{2}}$} & $\hat{a}[n]$ & $\operatorname{med}_{1=0}^{\mathrm{L}-1}\left(y_{l}\left[N_{p}+n\right]\right)$ & \multirow{3}{*}{$T_{L B}(\mathbf{X})=\frac{\hat{\sigma}_{0}}{\hat{\sigma}_{1}}$} \\
\hline & $\hat{\sigma}_{1}^{2}$ & $\frac{1}{N L} \sum_{n=0}^{N-1} \sum_{l=0}^{L-1}\left(x_{l}^{\prime}[n]-\hat{s}[n]\right)^{2}$ & & $\hat{\sigma}_{1}$ & $\frac{\sqrt{2}}{N L} \sum_{n=0}^{N-1} \sum_{l=0}^{L-1}\left|x_{l}^{\prime}[n]-\hat{s}[n]\right|$ & \\
\hline & $\hat{\sigma}_{0}^{2}$ & $\frac{1}{N L} \sum_{n=0}^{N-1} \sum_{l=0}^{L-1}\left(x_{l}^{\prime}[n]\right)^{2}$ & & $\hat{\sigma}_{0}$ & $\frac{\sqrt{2}}{N L} \sum_{n=0}^{N-1} \sum_{l=0}^{L-1}\left|x_{l}^{\prime}[n]\right|$ & \\
\hline \multirow{3}{*}{$\mathrm{C}$} & $\hat{a}[n]$ & $\frac{1}{L} \frac{\sum_{l=0}^{L-1} \frac{y_{l}\left[N_{p}+n\right]}{\hat{\sigma}_{l, 1}^{2}}}{\sum_{l=0}^{L-1} \frac{1}{\hat{\sigma}_{l, 1}^{2}}}$ & \multirow{3}{*}{$T_{G C}(\mathbf{X})=\prod_{l=0}^{L} \frac{\hat{\sigma}_{l, 0}^{2}}{\hat{\sigma}_{l, 1}^{2}}$} & $\hat{a}[n]$ & $\operatorname{med}_{1=0}^{\mathrm{L}-1}\left(y_{l}\left[N_{p}+n\right] \diamond \frac{1}{\hat{\sigma}_{l, 1}}\right)$ & \multirow{3}{*}{$T_{L C}(\mathbf{X})=\prod_{l=0} \frac{\hat{\sigma}_{l, 0}}{\hat{\sigma}_{l, 1}}$} \\
\hline & $\hat{\sigma}_{l, 1}^{2}$ & $\frac{1}{N} \sum_{n=0}\left(x_{l}^{\prime}[n]-\hat{s}[n]\right)^{2}$ & & $\hat{\sigma}_{l, 1}$ & $\frac{\sqrt{2}}{N} \sum_{n=0}\left|x_{l}^{\prime}[n]-\hat{s}[n]\right|$ & \\
\hline & $\hat{\sigma}_{l, 0}^{2}$ & $\frac{1}{N} \sum\left(x_{l}^{\prime}[n]\right)^{2}$ & & $\hat{\sigma}_{l, 0}$ & $\frac{\sqrt{2}}{N} \sum_{n=0}\left|x_{l}^{\prime}[n]\right|$ & \\
\hline
\end{tabular}

linear phase FIR low-pass filter with transition band between 15 and $30 \mathrm{~Hz}$ ), ST-T segmentation, alignment and decimation. The length of the analysis window was $L=32$ beats. As the location of the TWA episode is usually not known, the presented GLRT detectors are applied on a sliding-window basis, so that the test statistic is computed and compared with the threshold for each new beat.

\subsection{Simulation study}

To evaluate the TWA detectors under real noise conditions, we used records (Frank leads) from healthy subjects from the Politecnico-Ca' Granda Database [5]. We extracted 413 ECG segments (128 beats long) from the 23 records. TWA episodes were simulated by adding and subtracting alternatively a Hanning window to the ST-T complexes. The amplitude of this waveform was beatby-beat modulated by a 56-beats-long trapezoidal episode shape, remaining during 32 beats at its maximum value. The episodes were centered in each 128-beat segment and the simulated TWA amplitudes ranged from $0 \mu \mathrm{V}$ to $100 \mu \mathrm{V}$. The performance of the detectors for each TWA amplitude was evaluated in terms of episode sensitivity $S e$ and specificity $S p$ for a given threshold. Sweeping different threshold values, ROC curves were obtained.

\section{Results}

As the level of the noise present in the recordings was very different in the three leads, we show the results for lead
$\mathrm{X}$ (which was generally the one with least noise) and for lead Y (the noisiest one). Figure 1 shows the ROC curves for the Gaussian (a), (b) and Laplacian detectors (c), (d). We show the results for a TWA amplitude of $5 \mu \mathrm{V}$ in lead $\mathrm{X}$, and $20 \mu \mathrm{V}$ in lead $\mathrm{Y}$.

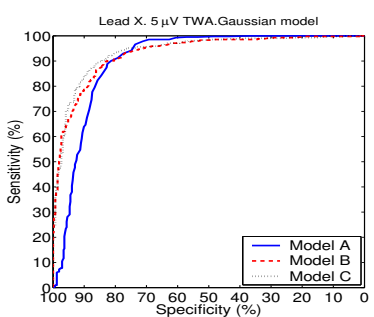

(a) Lead X, Gaussian model

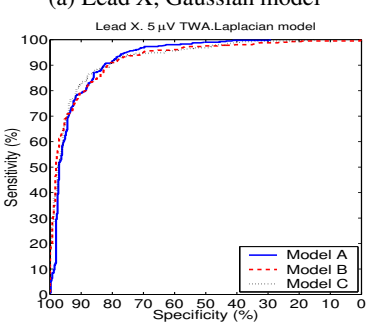

(c) Lead X, Laplacian model

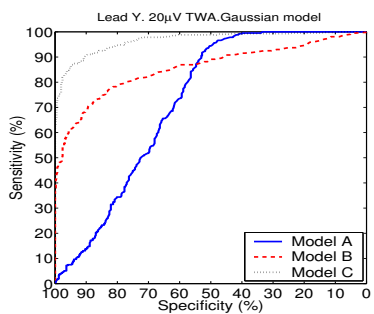

(b) Lead Y, Gaussian model

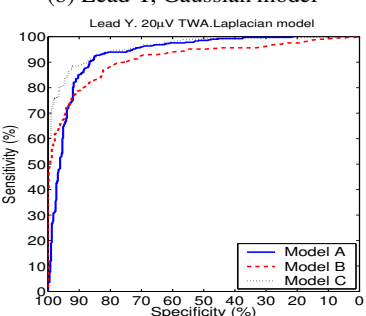

(d) Lead Y, Laplacian model
Figure 1. ROC curves in leads X (with TWA of $5 \mu \mathrm{V}$ ) and $\mathrm{Y}$ (TWA of $20 \mu \mathrm{V})$.

To compare in a simple way the behavior of the detectors for different TWA amplitudes, we summarize the information of the ROC curve with a single parameter, called $S 95$ and defined as the sensitivity (\%) of the detector using a threshold so that the specificity is $95 \%$. In Figure 2 
S95 is plotted against the TWA amplitude at leads X and Y for the six implemented detectors.

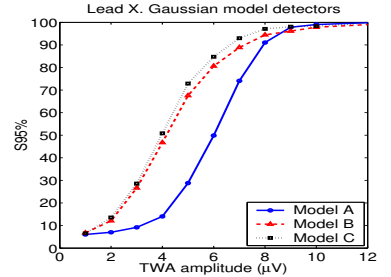

(a) Lead X, Gaussian model

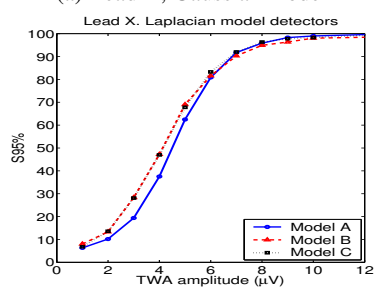

(c) Lead X, Laplacian model

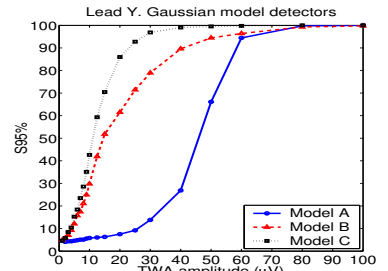

(b) Lead Y, Gaussian model

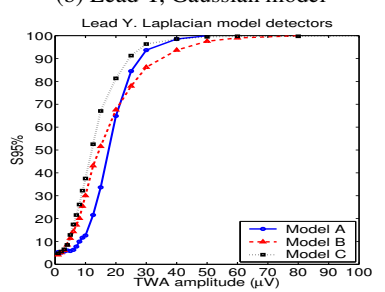

(d) Lead Y, Laplacian model
Figure 2. Performance of the detector $(S 95)$ as a function of the TWA amplitude.

\section{Discussion}

In low noise conditions (lead $\mathrm{X}$ ), the performance observed is similar for all the studied detectors, showing a slightly better performance the detectors based on nonstationary noise models as it can be seen in panels a) and c) of Figures 1 and 2.

When the noise level is important (lead Y), we found clearer differences among the detectors. In particular, under the assumption of stationary, known noise variance (Model A), the Laplacian detector outperforms clearly the Gaussian-based detector, supporting the hypothesis that real ECG noise affecting TWA detection can be better modeled with heavy-tailed distributions than with Gaussian distribution. However, when permitting the model to account for the noise variations, the differences between both probability densities vanishes, to the point that the Gaussian- and Laplacian-based detectors have essentially the same performance under Model C.

In the Gaussian case, the poor performance of the known stationary noise detector was significantly improved by using non-stationary models (see Figures 1(b) and 2(b)). In high noise conditions (lead Y), the TWA amplitude needed to attain $\mathrm{Se}=95 \%$ and $\mathrm{Sp}=95 \%$ was reduced from $62 \mu \mathrm{V}$ to $53 \mu \mathrm{V}$ using Detector 2 (which consists just on normalizing the stationary detector by the MLE estimator of the noise variance), and to $28 \mu \mathrm{V}$ using Model $\mathrm{C}$, which performs a beat-to-beat noise variance estimation.

However, the Laplacian-based detector shows a much slighter improvement. With the Laplacian models, even the simple Model A detector attains $\mathrm{Se}=95 \%$ and $\mathrm{Sp}=95 \%$ for amplitudes as low as $32 \mu \mathrm{V}$. Using Model $\mathrm{B}$, the performance is improved for low amplitude TWA $\left(V_{\text {alt }}<20\right.$ $\mu \mathrm{V})$, but it is degraded for higher amplitudes, probably due to the variance in the noise estimation, and the required $S e$ and $S p$ are only reached for $V_{\text {alt }}=43 \mu \mathrm{V}$. Finally, with Model C, the necessary amplitude drops to $28 \mu \mathrm{V}$.

\section{Conclusions}

In this work, we departed from the hypothesis that a good underlying noise model would improve the performance of a TWA detector. We derived GLRT detectors for Gaussian and Laplacian noise distributions as well as three different degrees of noise knowledge and stationarity. All models accounted, not only for the ST-T complex, but also for the $\mathrm{P}$ wave. The detectors were tested with real noise in ambulatory recordings with simulated TWA. The results of their evaluation highlight the importance of signal and noise modeling for designing a good TWA detector.

The results show that TWA detection performance in real noise conditions degrades when assuming a Gaussian stationary model. The beat-to-beat changing noise model has shown to be the most robust (but also the most complex), attaining the best performance for both the Gaussian- and Laplacian-based detectors. However, similar performance can also be achieved using a stationary Laplacian-based detector, which is intrinsically more robust to outliers.

\section{Acknowledgments}

This work was supported by projects TIC2001-2167C02-02 from the MCyT and FEDER and P075/2001 from DGA (Spain).

\section{References}

[1] Rosenbaum DS, Jackson LE, Smith JM, Garan H, Ruskin JN, Cohen RJ. Electrical alternans and vulnerability to ventricular arrhythmias. N Engl J Med January 1994;330(4):235-241.

[2] Nearing BD, Huang AH, Verrier RL. Dynamic tracking of cardiac vulnerability by complex demodulation of the $\mathrm{T}$ wave. Science 1991;252:437-440.

[3] Martínez JP, Olmos S. A robust T-wave alternans detector based on the GLRT for Laplacian noise distribution. In Computers in Cardiology 2002. IEEE, 2002; 677-680.

[4] Kay SM. Fundamentals of statistical signal processing, volume II-Detection theory. Prentice-Hall, 1998.

[5] Pinciroli F, Rossi R. ECG \& Resp. A biomedical signals database on CD-ROM from the Politecnico di Milano, 1992.

Address for correspondence:

Juan Pablo Martínez Cortés

Dep. Ingeniería Electrónica y Comunicaciones

Maria de Luna, 3. 50018-Zaragoza (Aragón). SPAIN

E-mail: jpmart@unizar.es 